\title{
Alveolar Hemorrhage Associated with Rivaroxaban: A Case Series
}

Heather Laird-Fick, Brian J Grondahı*, Megan Benedict and Anas Riehani

Department of Internal Medicine, Michigan State University, East Lansing, MI, USA

\begin{abstract}
Background: We present 4 cases of alveolar hemorrhage in patients receiving rivaroxaban. While bleeding is a common complication of rivaroxaban therapy reported by multiple case reports in the literature, Alveolar hemorrhage is not frequently reported. There were two cases of alveolar hemorrhage related to rivaroxaban found in our literature search. Alveolar hemorrhage is a potentially fatal complication and must be promptly recognized.
\end{abstract}

Case Presentation: The electronic medical record was reviewed for patients with alveolar hemorrhage syndromes admitted to our hospital between July 1, 2014 and June 30, 2015. This data was cross referenced for patients that received anticoagulant medications.

Our series of patients included one patient with underlying Antiphospholipid Syndrome (APS) and systemic lupus erythematous, another patient with pulmonary malignancy and distant lung resection, a third patient with lymphangioleiomyomatosis, and a fourth patient with myelodysplastic syndrome. Rivaroxaban accounted for $13.6 \%$ of all cases of clinically significant pulmonary hemorrhage and drug related pulmonary hemorrhage accounted for half of cases. The rate of alveolar hemorrhage associated with Rivaroxaban in our institution was about 3 cases in 1000.

Conclusions: We identified four cases of rivaroxaban associated alveolar hemorrhage. We encourage cautious use of rivaroxaban in patients with underlying lung conditions or factors that predispose to alveolar hemorrhage. Further research to clarify the risk of diffuse alveolar hemorrhage (DAH) in at-risk patients receiving oral anticoagulants is recommended.

Keywords: Rivaroxaban; Alveolar hemorrhage; Novel oral anticoagulant; Adverse event; Bleeding complication; Antiphospholipid syndrome; Lymphangioleiomyomatosis

Abbreviations: APS: Antiphospholipid Syndrome; DAH: Diffuse Alveolar Hemorrhage; EMR: Electronic Medical Record; g/ dl: Grams per Deciliter; GFR: Glomerular Filtration Rate; LAM: Lymphangioleiomyomatosis; $\mathrm{ml} / \mathrm{min}$ : Milliliters per Minute; SLE: Systemic Lupus Erythematosus

\section{Introduction}

Novel anticoagulants such as rivaroxaban, a direct factor $\mathrm{X}_{\mathrm{a}}$ inhibitor, have presented advancement in management of venous thromboembolism and atrial fibrillation. The most common complication of rivaroxaban treatment is bleeding with a relative risk (when compared to warfarin) of non-major bleeding of 0.99 and a relative risk for fatal bleeding of 0.48 [1]. Diffuse alveolar hemorrhage $(\mathrm{DAH})$ related to rivaroxaban has only been reported in two cases $[2,3]$. Other causes of DAH incude systemic vasculitides, connective tissue diseases, infections, toxins and drugs. Diagnosis is confirmed by bronchoscopy with bloody fluid return on irrigation. Data regarding the overall incidence of DAH were lacking. We present four cases (Table 1) of rivaroxaban associated alveolar hemorrhage. Alveolar hemorrhage should be considered in patients receiving anticoagulation who develop hemoptysis, respiratory failure, or bilateral ground glass opacities on chest X-ray.

\section{Case Presentation}

\section{Case 1}

The patient is a 45 -year-old woman with a past medical history of systemic lupus erythematosis (SLE) complicated by lupus nephritis, antiphospholipid syndrome (APS) with aortic and renal artery thrombosis, and autoimmune hemolytic anemia. There was no evidence of pulmonary involvement of SLE prior to presentation. Her renal function was stable with a glomerular filtration rate of $51 \mathrm{ml} /$ $\mathrm{min}$. She was prescribed $20 \mathrm{mg}$ of rivaroxaban daily for the preceding
19 days. She presented with complaints of dyspnea and hemoptysis. Her condition deteriorated quickly and she required mechanical ventilation. Chest imaging revealed bilateral opacification of the lungs. Laboratory studies showed an acute anemia with hemoglobin level of $5.8 \mathrm{~g} / \mathrm{dl}$ (baseline of approximately $8.5 \mathrm{~g} / \mathrm{dl}$ ). Bronchoscopy was performed and confirmed the diagnosis of DAH. Rivaroxaban was discontinued and she was treated with red blood cell transfusion, high dose steroids, and intra-pulmonary activated factor VII concentrate. Her renal function declined and it was felt that this was partly due to worsening lupus nephritis for which cyclophosphamide was started. The patient recovered and has been stable on follow up visits for several months. She was not restarted on oral anticoagulation as she developed hemoptysis with a challenge dose of rivaroxaban of $20 \mathrm{mg}$.

\section{Case 2}

This patient is an 80 -year-old man with a past medical history significant for adenocarcinoma of the lung (treated with partial left lobectomy two years prior), atrial fibrillation, chronic obstructive pulmonary disease, and chronic hypoxemic respiratory failure with tracheostomy. The patient was receiving rivaroxaban $20 \mathrm{mg}$ daily. He developed worsening hypoxia and hemoptysis and required mechanical ventilation. Chest radiograph revealed bilateral infiltrates. His hemoglobin decreased from $10.6 \mathrm{~g} / \mathrm{dl}$ to $7.4 \mathrm{~g} / \mathrm{dl}$, so rivaroxaban was discontinued. Bronchoscopy confirmed the diagnosis of DAH. The patient and family opted for a palliative care approach and he expired.

*Corresponding author: Brian J Grondahl, Department of Internal Medicine, Michigan State University, 788 Service Road, Room B301, East Lansing, MI, USA Tel: 5173535100; E mail: brian.grondahl@hc.msu.edu

Received April 17, 2017; Accepted May 09, 2017; Published May 13, 2017

Citation: Laird-Fick H, Grondahl BJ, Benedict M, Riehani A (2017) Alveolar Hemorrhage Associated with Rivaroxaban: A Case Series. J Pulm Respir Med 7: 405. doi: 10.4172/2161-105X.1000405

Copyright: (c) 2017 Laird-Fick H, et al. This is an open-access article distributed under the terms of the Creative Commons Attribution License, which permits unrestricted use, distribution, and reproduction in any medium, provided the original author and source are credited. 


\begin{tabular}{|c|c|c|c|c|c|c|c|c|c|}
\hline Case No. & Age & Sex & Medical history & GFR & Indication & \begin{tabular}{|c|} 
Duration of \\
Treatment (days)
\end{tabular} & $\begin{array}{l}\text { Baseline Hgb } \\
\text { (g/dl) }\end{array}$ & $\begin{array}{l}\text { Presenting Hgb } \\
\text { (g/dl) }\end{array}$ & $\begin{array}{c}\text { Hgb Change } \\
\text { (g/dl) }\end{array}$ \\
\hline Case 1 & 45 & $\mathrm{~F}$ & SLE Anti-phosholipid syndrome & 51 & Arterial thrombus & 19 & 8.5 & 5.8 & 2.7 \\
\hline Case 2 & 80 & M & $\begin{array}{c}\text { Stage } 1 \text { Adenocarcinoma of lungs } \\
\text { Pneumonia }\end{array}$ & 76 & Atrial Fibrillation & 90 & 10.6 & 7.4 & 3.2 \\
\hline Case 3 & 79 & $\mathrm{~F}$ & LAM & 83 & Pulmonary embolism & 13 & 11.7 & 6.4 & 5.3 \\
\hline Case 4 & 75 & $\mathrm{M}$ & MDS & 50 & Atrial Fibrillation & 84 & 8 & 6.2 & 1.8 \\
\hline & \multicolumn{5}{|c|}{ Average } & 51 & & & 3.25 \\
\hline
\end{tabular}

Table 1: Summary of alveolar hemorrhage cases

\section{Case 3}

This patient is a 79-year-old woman with history of lymphangioleiomyomatosis (LAM) and recent diagnosis of deep venous thrombosis and bilateral pulmonary embolism two weeks prior. She was treated with rivaroxaban $20 \mathrm{mg}$ daily. She presented with dyspnea, cough, and hemoptysis. Chest radiography showed bilateral infiltrates with a right-sided pleural effusion. Laboratory studies showed an acute anemia with fall in hemoglobin from $10.5 \mathrm{~g} / \mathrm{dl}$ on presentation to $7.6 \mathrm{~g} / \mathrm{dl}$ one day later. Her respiratory status declined and she required intubation and mechanical ventilation. Rivaroxaban was held and a thoracentesis was performed on a right-sided pleural effusion revealing grossly bloody fluid. A bronchoscopy was performed which confirmed the diagnosis of DAH. An inferior vena cava filter was placed, and the patient recovered with supportive treatment. She was discharged after fifteen days in the hospital. This patient was not restarted on anticoagulation and unfortunately was lost to follow-up.

\section{Case 4}

Probable alveolar hemorrhage was identified in a 75-year-old man with history of myelodysplastic syndrome (refractory anemia with excess blasts-2) that had progressed to acute myelogenous leukemia. $\mathrm{He}$ never received chemotherapy. The patient was receiving rivaroxaban $15 \mathrm{mg}$ once daily for a diagnosis of atrial fibrillation. His GFR was calculated at $50 \mathrm{ml} / \mathrm{min}$. He was admitted after a fall resulting in a subarachnoid hemorrhage. He developed hemoptysis and respiratory failure with bilateral ground glass opacification on chest imaging. His platelet count was $47,000 / \mu \mathrm{l}$ and hemoglobin was $6.2 \mathrm{~g} / \mathrm{dl}$, (baseline approximately $8 \mathrm{~g} / \mathrm{dl}$ ). He was transitioned to comfort-directed care and no further procedures were done to confirm a diagnosis of $\mathrm{DAH}^{*}$.

Two cases were reviewed from the literature. The first is of a patient with history of partial lobectomy for bronchiectasis. He was prescribed rivaroxaban and amiodarone and developed pulmonary hemorrhage after one year. He did well after discontinuation of the medication combination and was successfully restarted on rivaroxaban without amiodarone [3]. The other was a post-mortem report of a patient with interstitial lung disease who was given rivaroxaban for deep venous thrombosis prevention following total knee arthroplasty. Findings at autopsy revealed changes consistent with DAH [2].

\section{Methods}

We aimed to identify additional cases of anticoagulation-related DAH and the incidence thereof at our centre. We performed a search of our electronic medical record (EMR) during a one year time period of July 1, 2014 and June 30, 2015 for cases of alveolar haemorrhage including DAH, massive haemoptysis and pulmonary haemorrhage. This search returned 22 cases of significant alveolar haemorrhage that was not considered simple haemoptysis or blood-streaked sputum. In the same time period as the EMR search, we also queried the inpatient pharmacy for the number of unique patients rivaroxaban was dispensed to.

\section{Results}

Rivaroxaban accounted for $13.6 \%(n=4)$ of all cases of clinically significant pulmonary hemorrhage and drug related pulmonary hemorrhage accounted for half of cases.

There were additional cases of pulmonary hemorrhage on anticoagulants (one on dabigatran and two on warfarin). There were also cases of antiplatelet associated alveolar hemorrhage with one observed on prasugrel and three with clopidogrel. The remainder of the cases were accounted for by Wegener's granulomatosis (one), malignancy (six), infectious (three), and post-procedural (four) ${ }^{* *}$.

We found 1,060 unique individuals were dispensed Rivaroxaban, resulting in the rate of alveolar hemorrhage associated with Rivaroxaban of approximately 0.0028 to 0.0037 , or about 3 cases in 1,000 . The rate of DAH associated with warfarin was 0.0013 ( 2 cases in 1,561 patients given warfarin).

\section{Discussion}

Alveolar hemorrhage associated with rivaroxaban is an apparently rare complication. There were no reported cases of DAH in the ROCKET-AF study [1]. We identified two additional cases in the literature, both of which included patients with underlying lung conditions $[2,3]$. The patients identified in this case series all have underlying chronic medical conditions. Case one identified a woman with SLE and APS. APS is associated with alveolar hemorrhage, either as a primary etiology or in combination with other connective tissue disorders such as SLE. The incidence of DAH associated with APS is unknown, but 22 cases were presented in one series [4]. The patient was treated with Rivaroxaban for an arterial thrombosis, which is not an approved indication [5]. She also had a worsening renal function during hospitalization which might contribute to the decrease in the rivaroxaban clearance leading to higher level of the drug in the system. The patient in case two had no recognized risk factors for $\mathrm{DAH}$, however he was diagnosed with chronic lung disease, resected adenocarcinoma, recurrent infections, and chronic respiratory failure. In case three, we present a patient with lymphangioleimyomatosis. This condition exclusively affects females and its incidence is not well established, though it estimated that there are about 1,300 cases of LAM in North America [6]. LAM has an association with clinically insignificant bleeding on biopsy and we could identify only two cases of spontaneous alveolar hemorrhage in LAM $[7,8]$. Finally, the fourth patient had an underlying diagnosis of myelodysplastic syndrome. No reliable evidence exists regarding pulmonary hemorrhages in this condition in the absence of infection, chemotherapy, or bone marrow transplantation. Furthermore, he had thrombocytopenia which might also have increased the risk for developing alveolar hemorrhage.

Given the multiple comorbidities and risk factors for bleeding in the aforementioned cases, assessment of anticoagulation effect of rivaroxaban utilizing chromogenic anti- $\mathrm{X}_{\mathrm{a}}$ assays would have provided 
an estimate of rivaroxaban activity and possible clarification in regard to the culprit of DAH in those cases [9].

Diagnostically, the patients in this case series all developed acute dyspnea, bilateral infiltrates on chest X-ray and acute anemia with an average decrease in hemoglobin of $3.7 \mathrm{~g} / \mathrm{dl}$. The average length of time the patients were taking rivaroxaban was 41 days, and two patients were taking rivaroxaban less than three weeks. Bronchoscopy is still the gold standard test of choice to confirm the diagnosis of DAH, however, in the fourth case the patient passed away before preforming the bronchoscopy. Diagnosis can be made based on postpartum autopsy [10].

In one of our cases, activated factor VII was given during bronchoscopy and resulted in good hemostasis. This is a potential treatment for alveolar hemorrhage syndromes and was reported in series of patients previously [11].

\section{Conclusion}

In our study, we identify four cases of rivaroxaban associated alveolar hemorrhage. There is no definitive evidence that rivaroxaban was the direct cause of the hemorrhage, but we do believe that the temporal relationship and relative short length of time prior to the bleeding complication suggests correlation. We believe that this may be an under-recognized condition as multiple cases were identified in our institution, yet literature reports are rare. There are case reports of alveolar hemorrhage associated with nearly all anticoagulants, including warfarin [12], low molecular weight heparin [13], and apixaban [14]. The patients highlighted in this case series all had underlying conditions that may have predisposed them to the complication of alveolar hemorrhage. Strategies of reversing the novel anticoagulants are being researched; there remains no proven way to reverse all of these agents. Therefore, we believe that the novel anticoagulants should be used cautiously in this subset of patients with lung disease and conditions that may predispose to the potentially fatal complication of DAH. We suggest further study including post marketing surveillance of bleeding complications during anticoagulant therapy. A randomized controlled trial is not likely suitable in this case, but retrospective study can further clarify risk factors for alveolar hemorrhage.

*The Adverse Drug Reaction Probability Scale was applied to case 4 [15]. This tool resulted in a score of 5 and an adverse reaction related to rivaroxaban as "probable."

"Duplication of cases does exist, for example two of the post procedure cases were also in patients with lung cancer.

\section{Declarations}

Ethics approval and consent to participate: There has been approval from the Institutional Review Board at Michigan State University, IRB\#15-349M.

Consent for publication: Written informed consent to participate and publish has been obtained from the subjects presented in the cases, with the exception of deceased individuals as guided by the local IRB.

Availability of data and material: The datasets generated during and analyzed during the current study are not publicly available due to IRB restrictions, but are available from the corresponding author on reasonable request.

Funding: No funding was obtained to carry out this project.

Competing interest: We have no conflicts of interest to disclose

Author contribution: Heather Laird-Fick served as principal investigator and made contribution to study design, data analysis, and writing/editing responsibilities. Brian $\mathrm{J}$ Grondahl is the corresponding author and was responsible for a bulk of data analysis and manuscript writing. Megan Benedict served to obtain and write background information as well as the case summary for case 3. Anas Riehani contributed by reviewing literature cases and providing their case summaries and to writing case 2. All authors contributed to the final text and approved of the manuscript as presented.

\section{Acknowledgement}

We would like to acknowledge Denise Pratt, PharmD, for her assistance in gathering pharmacy data.

\section{References}

1. Patel MR, Mahaffey KW, Garg J, Pan G, Singer DE, et al. (2011) Rivaroxaban versus warfarin in nonvalvular atrial fibrillation. N Engl J Med 365 883-891.

2. Hammar SP (2015) Fatal pulmonary hemorrhage after taking an anticoagulant medication. Respir Med Case Rep 15: 66-70.

3. Elikowski W, Malek M, Skowronski M, Wróblewski D, Skrzywanek P, et al. (2015) Hemoptysis during concomitant treatment with rivaroxaban and amiodarone in a patient with a history of pulmonary disease. Pol Merkur Lekarski 39: 227-230.

4. Kazzaz NM, Coit P, Lewis EE, Emily EL, Joseph M, et al. (2015) Systemic lupus erythematosus complicated by diffuse alveolar haemorrhage: risk factors, therapy and survival. Lupus Sci Med 2: e000117.

5. Sciascia S, Lopez-Pedrera C, Cecchi I, Pecoraro C, Roccatello D, et al. (2016) Non-vitamin $\mathrm{K}$ antagonist oral anticoagulants and antiphospholipid syndrome. Rheumatology (Oxford) 55: 1726-1735.

6. Taveira-DaSilva AM, Moss J (2015) Clinical features, epidemiology, and therapy of lymphangiomyomatosis. Clin Epidemiol 7: 249-257.

7. Baldi BG, Pimenta SP, Kawassaki AM, Bernardi FDC, Dolhnikoff M, et al. (2011) Pulmonary arterial involvement leading to alveolar hemorrhage in lymphangioleiomyomatosis. Clinics (Sao Paulo) 66: 1301-1303

8. Yamamoto K, Anzai F, Kusajima K, Yamanishi N, Yamada M (2004) Acute respiratory failure with gross hemoptysis in a patient with lymphangioleiomyomatosis as part of tuberous sclerosis complex. Intern Med 43: 755-758.

9. Königsbrügge O, Quehenberger P, Belik S, Weigel G, Seger C, et al. (2015) Anti-coagulation assessment with prothrombin time and anti-Xa assays in realworld patients on treatment with rivaroxaban. Ann Hematol 94: 1463-1471.

10. Lichtenberger JP, Digumarthy SR, Abbott GF, Shepard JA, Sharma A (2014) Diffuse pulmonary hemorrhage: clues to the diagnosis. Curr Probl Diagn Radiol 43: $128-139$

11. Heslet $L$, Nielsen JD, Levi $M$, Sengeløv $H$, Johansson PI (2006) Successful pulmonary administration of activated recombinant factor VII in diffuse alveolar hemorrhage. Crit Care 10: R177.

12. Erdogan D, Kocaman O, Oflaz H, Goren T (2004) Alveolar hemorrhage associated with warfarin therapy: a case report and literature review. Int J Cardiovasc Imaging 20: 155-159.

13. Shinichi H, Shuichiro M, Yoshiko N, Noriaki T, Shu H (2013) Diffuse alveolar hemorrhage associated with low molecular weight heparin. Am J Cardiol 112 454-460.

14. Gabrilovich MI, Buxton DE, Lykins DM, McMillen SM, Onadeko OO (2014) Diffuse alveolar hemorrhage secondary to apixiban administration. Chest 146 : e115-e116.

15. Naranjo CA, Busto U, Sellers EM, Sandor P, Ruiz I, et al. (1981) A method for estimating the probability of adverse drug reactions. Clin Pharacol Ther 30 : $239-245$. 